\title{
ERRATA IN THE LEHMER FACTOR STENCILS
}

BY J. D. ELDER

In his factor stencils, D. N. Lehmer has made available a powerful tool in factorization problems. However, they present in use certain minor disadvantages. It was suggested to me that possibly these could be eliminated by using Hollerith cards. An additional advantage would be the ease of reproduction. I constructed with these cards a small set of factor stencils equivalent to the first eight columns of the Lehmer stencils. (As first constructed these were merely a partial copy of the Lehmer stencils.) These were so perfect mechanically that a thorough comparison of different stencils was extremely easy. A comparison of the new stencils for $+R$ and $-R$, for various prime values of $R$ showed contradictions, and further investigations convinced me of the desirability of a complete recalculation of the Lehmer stencils.

This is now well under way and has been completed for the first eight columns. The number of errors found is larger than anticipated. It seems desirable to make this information available, as it will take the better part of a year to complete the entire calculation. Professor Lehmer has graciously loaned the original stencils to me for purposes of comparison; in a few entries these differ from the copy which was first used.

In the following table the number in the $L$-column indicates the number of the cell considered; $\mathrm{a}+$ or - indicates, respectively, that the cell in question is or is not punched. $C$ refers to the copy of Lehmer's stencil at the University of Michigan, $O$ to the original stencil, and $K$ to the correct entries. The entry for $C$ is given only if it differs from the corresponding $O$ entry.

A comparison of the following table and the original stencils disclosed the significant fact that almost all of the errors found had been made after the direct calculation for prime values of $R$ was completed. I had replaced Professor Lehmer's visual method of constructing stencils for composite $R$ 's by a photographic method which eliminates some of the possible sources of error; it has the additional advantage of requiring considerably less time. 


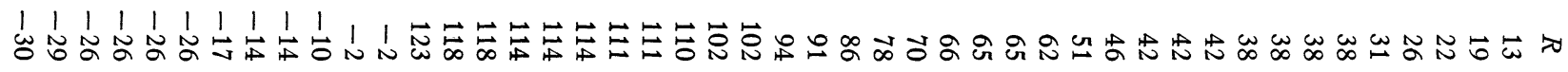

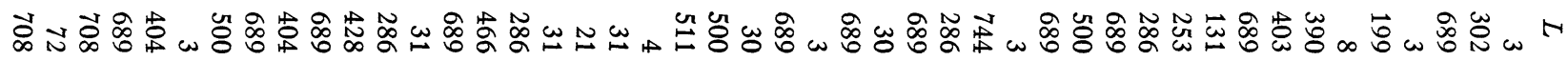
$++++++++++++1++1++++++++++++++1+++++++++++1+++\pi$ 11

$1111111111+++11+1111111111111111+11111111111+1+10$

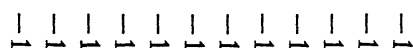

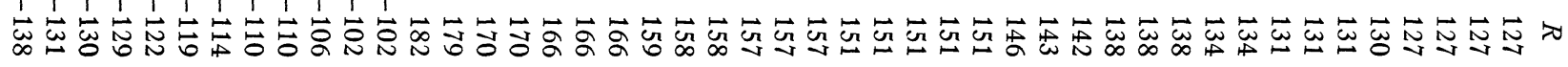

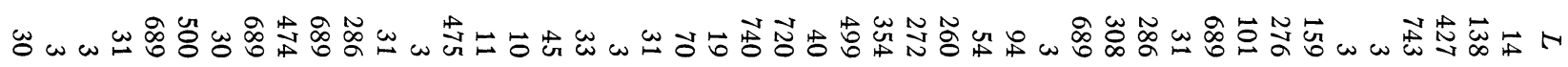
$++1+++++++++1++++++1++11+++++++1++1+++++1+++++\infty$

$11+1|1| 1|1| 1 \mid++11111+11++11111111+1++111111+111110$

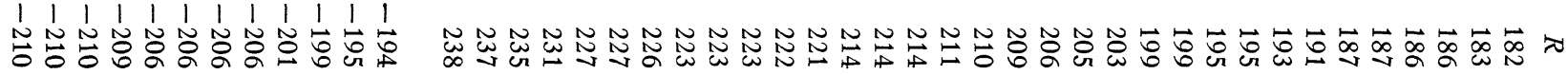

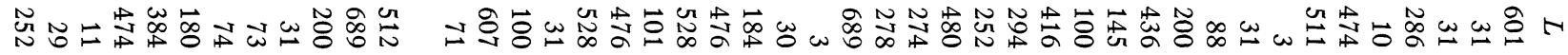
$+++++++++++++++1+++++++1+++++++1+++++++++111+\approx$ 


\begin{tabular}{|c|c|c|c|c|c|c|c|c|c|c|c|c|c|}
\hline$R$ & $L$ & $K$ & $C$ & $O$ & $R$ & $L$ & $K$ & $C$ & $O$ & $R$ & $L$ & $K$ & $C$ \\
\hline-34 & 500 & + & & - & -141 & 31 & + & & - & -213 & 31 & + & \\
\hline 35 & 101 & + & & - & -141 & 688 & + & - & + & -214 & 100 & + & \\
\hline-37 & 3 & - & & + & -142 & 576 & + & - & + & -214 & 176 & + & \\
\hline-38 & 303 & + & & - & -143 & 3 & + & & - & -215 & 100 & + & \\
\hline-39 & 509 & + & & - & -157 & 720 & - & & + & -215 & 161 & t & \\
\hline-41 & 101 & + & & - & -157 & 740 & + & & - & -218 & 689 & + & \\
\hline-62 & 13 & + & & - & -158 & 19 & + & & - & -221 & 3 & + & \\
\hline-65 & 3 & + & & - & -165 & 31 & + & & - & -221 & 476 & t & \\
\hline-66 & 30 & + & & - & -166 & 45 & + & & - & -221 & 500 & + & \\
\hline-69 & 42 & + & & - & -166 & 147 & + & & - & -221 & 528 & + & \\
\hline-74 & 4 & + & & - & -170 & 500 & + & & - & -222 & 31 & + & \\
\hline-74 & 689 & + & & - & -170 & 689 & + & & - & -222 & 100 & + & \\
\hline-77 & 27 & + & & - & -173 & 46 & + & - & + & -222 & 286 & + & \\
\hline-78 & 31 & - & & + & -174 & 31 & + & & - & -226 & 101 & + & \\
\hline-78 & 286 & + & & - & -174 & 286 & + & & - & -229 & 528 & + & \\
\hline-85 & 500 & + & & - & -177 & 31 & + & & - & -231 & 31 & - & \\
\hline-86 & 73 & + & & - & -181 & 45 & + & & - & -235 & 161 & + & \\
\hline-86 & 86 & + & & - & -182 & 3 & + & & - & -237 & 31 & + & \\
\hline-91 & 3 & - & & + & -187 & 500 & + & & - & -238 & 500 & + & \\
\hline-93 & 31 & $\overline{-}$ & & + & -190 & 11 & + & & - & -238 & 689 & + & \\
\hline-93 & 184 & + & & - & -193 & 3 & - & & + & & & & \\
\hline
\end{tabular}

Note by the Editors. This table has been examined by D. N. Lehmer and is thought by him to be correct. It has been re-checked by the author independently from the galley proofs, so that there should be no typographical errors. THE EDITORs.

UniverstTy OF Michigan 